\title{
Parameter Identification and State-of-Charge Estimation for Li-Ion Batteries Using an Improved Tree Seed Algorithm
}

\author{
Weijie CHEN ${ }^{\dagger}$, Ming $\mathrm{CAI}^{\dagger}$, Xiaojun TAN ${ }^{\dagger a)}$, Nonmembers, and $\mathrm{Bo} \mathrm{WEI}^{\dagger \dagger}$, Student Member
}

\begin{abstract}
SUMMARY Accurate estimation of the state-of-charge is a crucial need for the battery, which is the most important power source in electric vehicles. To achieve better estimation result, an accurate battery model with optimum parameters is required. In this paper, a gradient-free optimization technique, namely tree seed algorithm (TSA), is utilized to identify specific parameters of the battery model. In order to strengthen the search ability of TSA and obtain more quality results, the original algorithm is improved. On one hand, the DE/rand/2/bin mechanism is employed to maintain the colony diversity, by generating mutant individuals in each time step. On the other hand, the control parameter in the algorithm is adaptively updated during the searching process, to achieve a better balance between the exploitation and exploration capabilities. The battery state-of-charge can be estimated simultaneously by regarding it as one of the parameters. Experiments under different dynamic profiles show that the proposed method can provide reliable and accurate estimation results. The performance of conventional algorithms, such as genetic algorithm and extended Kalman filter, are also compared to demonstrate the superiority of the proposed method in terms of accuracy and robustness.

key words: parameter identification, optimizing algorithm, tree seed algorithm, differential evolution, swarm intelligence
\end{abstract}

\section{Introduction}

Interest in electric vehicles (EVs) has considerably increased in recent years due to their lower greenhouse gas emission and less energy consumption. Compared with other existing rechargeable batteries, Li-ion batteries are the most promising candidate to meet the range and lifetime demands of the vehicles. In practical applications, the single battery cells should be integrated to form a battery pack or system and be controlled by the battery management system (BMS) to ensure safe and efficient operation [1].

One of the most important functions of BMS is to estimate the state-of-charge (SOC) of the battery [2]. As a crucial indicator reflecting the remaining capacity of the battery, the SOC should be accurately provided to achieve optimal performance, predict a reliable operating time and avoid over-charging or over-discharging. However, it is unfeasible to measure the SOC directly, and thus a proper estimation method is needed.

Great effort has been exercised to provide accurate

Manuscript received January 13, 2019.

Manuscript revised April 2, 2019.

Manuscript publicized May 17, 2019.

${ }^{\dagger}$ The authors are with the School of Intelligent Systems Engineering, Sun Yat-sen University, China.

${ }^{\dagger}$ The author is with the Graduate School of Fundamental Science and Engineering, Waseda University, Tokyo, 169-8555 Japan.

a) E-mail: tanxj@mail.sysu.edu.cn (Corresponding author) DOI: 10.1587/transinf.2019EDP7015
SOC estimation in the literature. Among all the methods, the most intuitive way is to completely discharge the battery and obtain the amount of charge in the battery by recording discharged ampere-hours [3], [4]. A major problem with such method is that it may interrupt the normal operation of the battery, and meanwhile the discharging time is relatively long. The Coulomb counting method calculates the remaining charge by integrating the current flow over time, and it is widely used due to its conceptual simplicity and applicability. However, the accuracy of Coulomb counting is vulnerable to the initial SOC value, the Coulomb efficiency, as well as the accumulated error due to inaccurate sensors or low sampling rate [5]-[7]. The open circuit voltage (OCV) method determines the SOC by searching the predetermined OCV-SOC table [8]-[10]. The main problem with this method is that the OCV-SOC relationship may change with temperature and state-of-health $(\mathrm{SOH})$ of the battery, and thus large uncertainty could be generated.

Many advanced methods have been proposed in the literature to improve SOC estimation performance, which can be generally classified into two categories: the blackbox model based methods and the state-space model based methods [4], [11], [12]. The black-box models, such as neural networks [13]-[15], fuzzy logic [16]-[19] and supporting vector regression [20]-[22], are able to establish the implicit inherent relation between system input and output, and are widely used for different tasks, such as pattern classification, data verification and system modeling with contradictory inputs. Such methods are also proved to be effective for battery SOC estimation. However, it is difficult to obtain sufficient data for all different situations and the estimation result could be highly imprecise in the region where training data is not enough. On the other hand, estimation approaches combining the state-space model with Kalman filter or other observers [23]-[25] have been far investigated in recent years. The extended Kalman filter is proposed for SOC estimation in [26] and is improved in [27]. However, such algorithms are strongly dependent on the accuracy of battery model. An improper model structure or parameter may result in remarkable estimation errors. In practical applications, the model structure is probably unchanged, and thus the parameter identification plays a critical role in maintenance of model accuracy. Furthermore, since the model parameters may change with different operating environments and aging conditions, an online identification technique is needed to estimate the parameters in real time. 
The pulse discharging [28] and electrochemical impedance spectroscopy [29], [30] are the most pervasive methods for parameter identification, which are only suitable for offline use. For online estimation, recursive least squares (RLS) [31], [32] and EKF [26], [33] have received considerable attention due to their ability to update parameters adaptively. Such methods are easy to implement and the parameters can be deduced in real time. Moreover, model parameters and state variables, such as $\mathrm{SOC}$ or $\mathrm{SOH}$, can be estimated simultaneously by comprising two filters running in parallel [8], [26]. However, uncertainty may be accumulated during the estimation process. If any inaccuracies exist in the parameters, those errors may be transferred to the state estimation, and vice versa. The propagation of the error may lead to system instability or even divergence. Furthermore, the recursive methods will take relatively long time to converge, which reduces the robustness of the methods. Different from the methods above, the parameter identification of battery is regarded as an optimization problem in this paper. An optimization problem is to find the best solution under certain conditions, and may be encountered in many applications, such as traffic signal timing, route decision, and congestion pricing strategy [34]-[36]. In order to solve the optimization problem, some general global optimizing algorithms, such as genetic algorithm (GA) [37], [38] particle swarm optimization (PSO) [39], [40] and ant colony optimization [41], [42] can be introduced to parameter identification. However, such methods are often used for offline applications. In contrary, we use a modified algorithm for online identification. Besides, the battery SOC is also incorporated into the parameters to be identified to achieve joint estimation.

In this paper, the parameter identification method based on an improved tree seed algorithm (TSA) is proposed and used for joint estimation with system states. The battery parameters and the SOC can be estimated simultaneously with improved accuracy and efficiency. The remainder of this paper is organized as follows. In Sect. 2, the equivalent circuit model used for parameter identification and SOC estimation is described. The corresponding state-space functions of the model are also deduced. In Sect. 3 , the principle of tree seed algorithm is presented and its update rules are improved to enhance the exploitation and exploration capabilities. The experimental configurations for acquiring testing data are introduced in Sect. 4. Then, the TSA-based parameter identification results are illustrated and are compared with GA in Sect. 5. The SOC estimation results and the comparison with fixed parameter EKF is also presented. Finally, the conclusion is drawn in Sect. 6.

\section{Battery Modeling and Parameter Identification}

\subsection{Description of Equivalent Circuit Model}

The battery models have been studied especially for the purpose of vehicle power control and battery states estimation. Comparing with other kinds of models, the equivalent

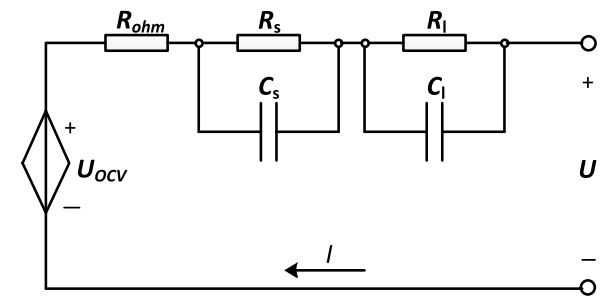

Fig. 1 Schematic diagram of the second-order RC model.

circuit models are widely accepted for practical applications due to their less parameters and lower computation burdens. Considering the balance of accuracy and complexity of the model, the second-order RC model is selected in this paper and is shown in Fig. 1, which includes a controlled voltage source, an ohmic resistance and two pairs of RC networks in series.

As depicted in Fig. 1, the battery dynamic and static characteristics can be simulated by the combination of several components. The controlled voltage source represents the open circuit voltage of the battery at different SOC levels. The two RC networks denote the time-dependent polarization and diffusion effects. And the ohmic resistance is used to describe the instant voltage drop after an excitation current in the battery.

\subsection{Parameter Identification}

Based on the battery model above, the parameters to be identified include: the ohmic resistance $R_{o h m}$, the polarization resistances $R_{s}$ and $R_{l}$, and the polarization capacitances $C_{s}$ and $C_{l}$. To achieve joint estimation of parameters and the state, the battery SOC is also integrated into the parameter vector.

The dynamic equations that describe the voltages across different parts of the model are given as:

$$
\begin{aligned}
& U_{\text {ohm }}=I R_{\text {ohm }} \\
& C_{s} \frac{d U_{s}}{d t}+\frac{U_{s}}{R_{s}}=I \\
& C_{l} \frac{d U_{l}}{d t}+\frac{U_{l}}{R_{l}}=I \\
& U=U_{o c v}-U_{\text {ohm }}-U_{s}-U_{l}
\end{aligned}
$$

where $U$ denotes the battery terminal voltage, $I$ denotes the battery current, and $U_{o c v}$ is the open circuit voltage. $R_{o h m}$, $R_{s}, R_{l}, C_{s}, C_{l}$ are the battery parameters which reflect the battery dynamic responses and are needed to be identified. $U_{\text {ohm }}$ is the voltage drop on $R_{\text {ohm }}$ and $U_{s}, U_{l}$ are the voltages across the two RC networks.

For further analysis, the discretization is applied to the system and one can acquire:

$$
\begin{aligned}
& U_{k}^{\text {ohm }}=I_{k} R_{\text {ohm }} \\
& U_{k}^{s}=I_{k} \frac{R_{s}}{1+R_{s} C_{s}}+\frac{R_{s} C_{s}}{1+R_{s} C_{s}} U_{k-1}^{s} \\
& U_{k}^{l}=I_{k} \frac{R_{l}}{1+R_{l} C_{l}}+\frac{R_{l} C_{l}}{1+R_{l} C_{l}} U_{k-1}^{l}
\end{aligned}
$$




$$
U_{k}=U_{k}^{o c v}-U_{k}^{o h m}-U_{k}^{s}-U_{k}^{l}
$$

where the subscript $k$ denotes the time step. For computational efficiency, the nonlinear OCV-SOC function within a relatively short period of time can be linearized as:

$$
U_{k}^{o c v}=a_{k} S O C_{k}+b_{k}
$$

where $a$ and $b$ are the linear coefficients.

According to the definition of SOC:

$$
S O C_{k}=S O C_{k-1}-I_{k} \frac{\eta_{k}}{C_{N}}
$$

where $C_{N}$ refers to the nominal capacity of the battery. To be simplified, the time interval is chosen as $1 \mathrm{~s}$ and the Coulomb efficiency $\eta_{k}$ is selected as 1 in this paper.

Define:

$$
\begin{aligned}
x_{k} & =\left[\begin{array}{llll}
U_{k}^{\text {ohm }} & U_{k}^{s} & U_{k}^{l} & \text { SOC }_{k}
\end{array}\right]^{T} \\
y_{k} & =U_{k} \\
A & =\left[\begin{array}{cccr}
0 & 0 & 0 & 0 \\
0 & \frac{R_{s} C_{s}}{1+R_{s} C_{s}} & 0 & 0 \\
0 & 0 & \frac{R_{l} C_{l}}{1+R_{l} C_{l}} & 0 \\
0 & 0 & 0 & 1
\end{array}\right] \\
B & =\left[\begin{array}{llll}
R_{\text {ohm }} & \frac{R_{s}}{1+R_{S} C_{s}} & \frac{R_{l}}{1+R_{l} C_{l}} & -\frac{1}{C_{N}}
\end{array}\right]^{T} \\
C & =\left[\begin{array}{llll}
-1 & -1 & -1 & a_{k}
\end{array}\right] \\
D & =\left[\begin{array}{llll}
0 & 0 & 0 & b_{k}
\end{array}\right]
\end{aligned}
$$
form:

Then Eq. (5)-Eq. (8) can be rewritten in a state-space

$$
\begin{aligned}
& x_{k}=A x_{k-1}+B I_{k} \\
& y_{k}=C x_{k}+D
\end{aligned}
$$

In the state-space model, the current $I$ is assumed as the input of the system and the terminal voltage $y$ is the output. The parameters in the system may intensively influence the input-output relationship and thus should be carefully identified. The optimum parameters can be determined by minimizing the root mean squared error between the predicted and the measured output. Then the objective function can be defined as:

$$
Z=\sqrt{\frac{1}{N} \sum_{k=1}^{N}\left(y_{k}-v_{k}\right)^{2}}
$$

where $y_{k}$ is the predicted voltage of the model at time step $k$ and $v_{k}$ is the measured voltage. $N$ denotes the number of samples. Then the heuristic methods, such as tree seed algorithm, can be used to minimize the function output and obtain the optimum model parameters.

\section{Tree Seed Algorithm}

To reduce the probability of being trapped into local minimum and acquire more accurate results, a method with global searching capability is required in the present work. Compared with the commonly used genetic algorithm, the suggested tree seed algorithm has fewer parameters and stronger searching ability, and thus it is more suitable for parameter identification. The algorithm is proposed based on the natural phenomena of trees and their seeds [43]. The locations of trees and seeds are regarded as possible solutions of the problem and will be updated in every generation, based on certain rules. The exploration and exploitation capabilities of the method can be well balanced by properly setting the control parameter. After a certain number of iterations, those possible solutions will gradually converge to the optimum for the problem.

In tree seed algorithm, the solution update rules are given as:

$$
\begin{aligned}
& S_{i, j, s}=T_{i, j}+\alpha_{i, j} \times\left(B_{j}-T_{r, j}\right) \\
& S_{i, j, s}=T_{i, j}+\alpha_{i, j} \times\left(T_{i, j}-T_{r, j}\right)
\end{aligned}
$$

where $S_{i, j, s}$ is the $j$ th dimension of the sth seed that generated by the $i$ th tree. $T_{i, j}$ is the $j$ th dimension of $i$ th tree. $B_{j}$ is the $j$ th dimension of the best-so-far tree location. $T_{r, j}$ is the $j$ th dimension of the $r$ th tree, which is randomly picked from the whole colony. $\alpha$ is the scaling factor arbitrarily produced in range of $[-1,1]$ and $i, j, s, r$ are the different indices.

At each time step, the seeds are generated either by Eq. (20) or Eq. (21), according to the result of comparing a random number and a control parameter named search tendency $(S T)$. If the randomly produced number is less than $S T$, then Eq. (20) is used, otherwise Eq. (21) is adopted.

In the beginning of operation with TSA, the tree locations, which represent the possible solutions for the optimization problem, are initialized by Eq. (22):

$$
T_{i, j}=L_{j, \text { min }}+r_{i, j} \times\left(H_{j, \text { max }}-L_{j, \text { min }}\right)
$$

where $L_{j, \min }$ is the lower bound of the search space of the $j$ th dimension and $H_{j, \max }$ is the upper bound. $r_{i, j}$ is a random number produced for every dimension and location, in range of $[0,1]$. In each generation, the number of seeds is randomly chosen between $10 \%$ and $25 \%$ of the colony size.

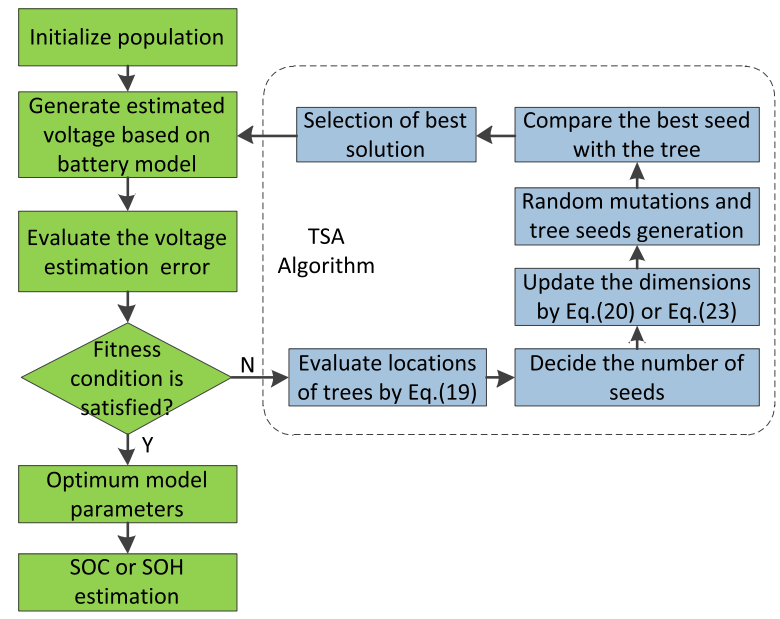

Fig. 2 Flow chart of the improved tree seed algorithm for parameter identification. 
In the original TSA, the balance of exploitation and exploration capabilities of the algorithm is controlled by the hyper parameter $S T$. The higher value of $S T$ offers a powerful local search and speed convergence while the lower value of $S T$ renders slow convergence but strong global search. However, the value of $S T$ is assumed to be fixed in the original algorithm and the controlling capability is not fully addressed. To enhance the estimation accuracy of the problem, the original TSA should be improved. Inspired by artificial bee colony algorithm [44], two adjustments are introduced to the origin TSA in this paper.

Firstly, the DE/rand/2/bin mechanism, which is proven to have better capability to maintain colony diversity, is applied to the algorithm to mutate every dimension of the seed. Then Eq. (21) in the original algorithm can be replaced by:

$$
S_{i, j, s}=T_{i 1, j}+F_{1}\left(T_{i 2, j}-T_{i 3, j}\right)+F_{2}\left(T_{i 4, j}-T_{i 5, j}\right)
$$

where $T_{i 1}, T_{i 2}, T_{i 3}, T_{i 4}$ and $T_{i 5}$ represent five randomly selected trees from the colony, $j$ denotes an arbitrary dimension of the possible solution, and $F_{1}, F_{2}$ are the scanning factors, which both equal to 0.5 .

Secondly, the control parameter $S T$ is vital for the whole searching process, but it is just a simple random number in the original algorithm. During the early iterations, the diversity of the algorithm should be emphasized while in the later cycles, the local search capability is demanded. Thus the $S T$ can be defined as:

$$
S T=0.1+0.9 *(\text { cycle } / \text { Maxcycle })^{2}
$$

where cycle denotes the current iteration step and Maxcycle represents the maximum cycle number. The general framework of parameter identification using improved TSA is shown in Fig. 2. The proposed method combines the exploration of differential evolution with the exploitation of the original TSA, and thus it is more effective for parameter identification.

\section{Experimental Configuration}

In order to verify and evaluate the applicability of the proposed parameter identification and SOC estimation algorithm, a test bench is built and several experiments are conducted, as shown in Fig. 3. The test bench consists of an Arbin battery testing system BT2000 for voltammetry measurement, a programmable thermal chamber for temperature control and a host computer for experimental control and data record. The cylindrical Li-ion cell from LG (LGDBMG11865) with nominal capacity of 2.9Ah and nominal voltage of $3.7 \mathrm{~V}$ is used for the tests.

In the experiment schedule, the available capacity test, the hybrid pulse power characterization (HPPC) test, the dynamic stress test (DST) and the federal urban dynamic schedule (FUDS) are consecutively conducted to the cell. The capacity test is used to measure the maximum capacity according to the method described in [4], [45]. The HPPC [11], [46] is used to obtain the OCV-SOC curve and the offline parameter table for some SOC estimation algorithms, such as EKF. The DST and FUDS are used to evaluate the accuracy of model parameters and SOC estimation results. The tests are conducted in the thermal chamber with constant temperature at $25^{\circ} \mathrm{C}$ and the data is recorded at $10 \mathrm{~Hz}$.

During the capacity test, the cell is firstly fully charged with constant current constant voltage mode, where the constant current is at $\mathrm{C} / 3$ rate, the constant voltage is the

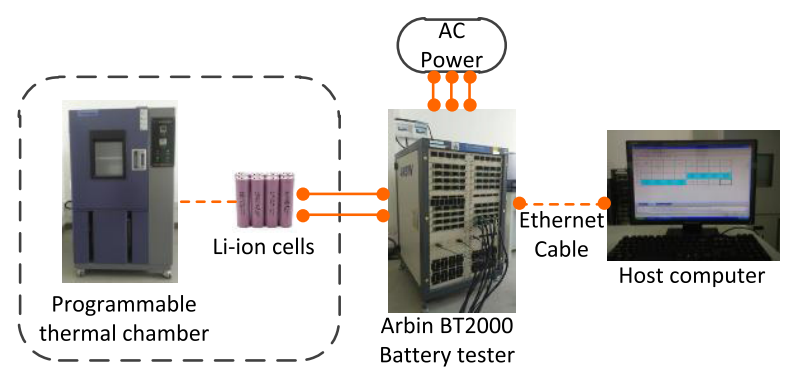

Fig. 3 The configuration of the battery test bench.

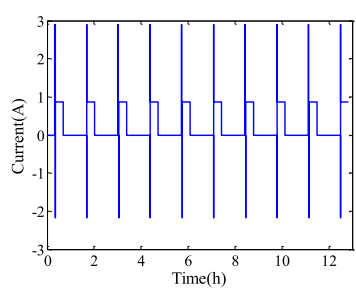

(a)

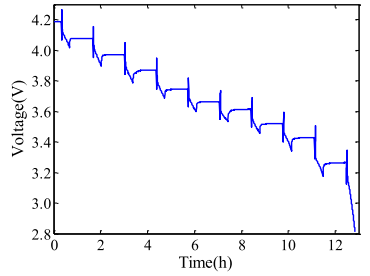

(b)
Fig. 4 Current and voltage profiles of HPPC test: (a) the current profile; (b) the voltage profile.

Table 1 Offline parameters obtained from HPPC test.

\begin{tabular}{cccccc}
\hline SOC & 0.1 & 0.2 & 0.3 & 0.4 & 0.5 \\
\hline OCV $(\mathrm{V})$ & 3.265 & 3.430 & 3.520 & 3.615 & 3.663 \\
$\mathrm{R}_{\text {ohm }}(\Omega)$ & 0.035 & 0.036 & 0.032 & 0.032 & 0.032 \\
$\mathrm{R}_{\mathrm{s}}(\Omega)$ & 0.046 & 0.045 & 0.029 & 0.038 & 0.034 \\
$\mathrm{C}_{\mathrm{s}}(\mathrm{F})$ & 1867.942 & 1873.687 & 3070.109 & 2954.636 & 2455.072 \\
$\mathrm{R}_{\mathrm{l}}(\Omega)$ & 0.019 & 0.016 & 0.008 & 0.019 & 0.009 \\
$\mathrm{C}_{\mathrm{l}}(\mathrm{F})$ & 63373.635 & 60452.240 & 90254.848 & 40305.776 & 81493.682 \\
\hline $\mathrm{SOC}$ & 0.6 & 0.7 & 0.8 & 0.9 & 1.0 \\
\hline $\mathrm{OCV}(\mathrm{V})$ & 3.747 & 3.872 & 3.971 & 4.078 & 4.186 \\
$\mathrm{R}_{\mathrm{ohm}}(\Omega)$ & 0.031 & 0.033 & 0.033 & 0.034 & 0.034 \\
$\mathrm{R}_{\mathrm{s}}(\Omega)$ & 0.026 & 0.020 & 0.041 & 0.028 & 0.027 \\
$\mathrm{C}_{\mathrm{s}}(\mathrm{F})$ & 2335.512 & 1478.341 & 1652.009 & 1627.797 & 1378.178 \\
$\mathrm{R}_{\mathrm{l}}(\Omega)$ & 0.013 & 0.044 & 0.010 & 0.006 & 0.003 \\
$\mathrm{C}_{\mathrm{l}}(\mathrm{F})$ & 68040.680 & 12166.235 & 34720.836 & 69915.624 & 15287.574 \\
\hline
\end{tabular}


upper limit voltage of the cell and the cut off current is $\mathrm{C} / 20$. After charge, the cell is stabilized for $1 \mathrm{~h}$ and then be discharged with $\mathrm{C} / 3$ current until the lower limit voltage is reached. The capacity test shows that the actual maximum capacity of the cell is $2.906 \mathrm{Ah}$, which is slightly larger than its nominal capacity.

During the HPPC test, the cell is firstly charged and rested in the same way as with the capacity test. Then the cell goes through ten hybrid pulse power cycles. Each cycle includes: a 10s-discharge, a 40s-rest, a 10s-charge, a 20 min-discharge at $\mathrm{C} / 3$ to decrease the cell SOC by $10 \%$, and finally a $1 \mathrm{~h}$-rest.

The voltage measured at the end of the rest is assumed to be the OCV of that SOC point. The current and voltage profiles of the HPPC test are shown in Fig. 4. The offline parameters can be obtained from the test and are shown in Table 1. As stated previously, the offline parameters are unable to track the characteristic changes of the battery, and thus should be optimized in real time.

\section{Verification and Comparison}

This section presents the verification results of the proposed method of battery parameter identification and SOC estimation. With regard to parameter identification, the model ac- curacy is evaluated by analyzing the discrepancy between the measured voltage and the predicted voltage, where the predicted voltage can be generated according to Eq. (11)Eq. (18). For comparison, three groups of voltage prediction data are generated by the improved TSA, GA and classical offline approach, respectively.

For SOC estimation, the performances of different algorithms are indicated by the estimation errors. The results of GA and EKF are presented for comparison. To verify the robustness of the algorithm, the input currents are added by a drift noise. The evaluations are conducted under DST and FUDS profiles. Moreover, the statistics describing the voltage discrepancy, such as the maximum absolute error (MAE) and the root mean squared error (RMSE), are listed for further analysis.

The current and voltage profiles of DST test are shown in Fig. 5 (a). Here a 1000s-segment is extracted from the whole test for detailed comparison. The current is positive for discharge in the figure and negative for charge. For the online methods, such as the improved TSA and GA, the parameters are estimated in real time using the given 1000 s data. For the offline approach, the parameters are obtained by searching the predetermined lookup table (Table 1). The estimated and measured voltages are demonstrated in Fig. 5 (b) and the estimation errors are shown in
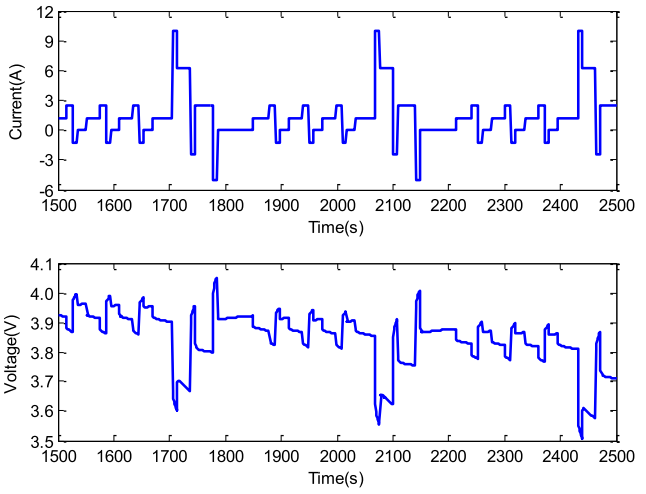

(a)

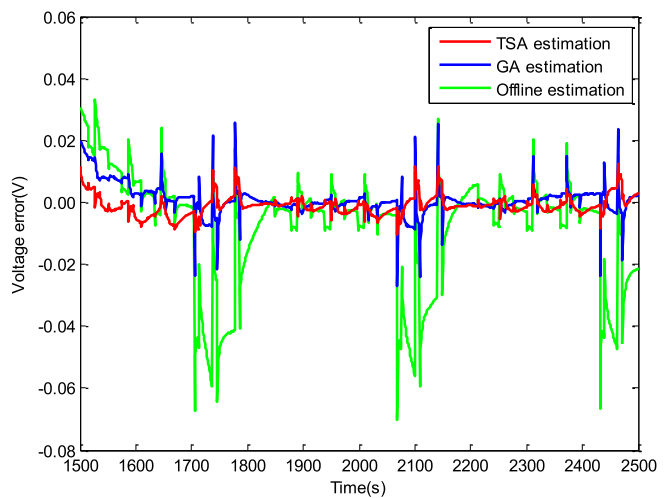

(c)

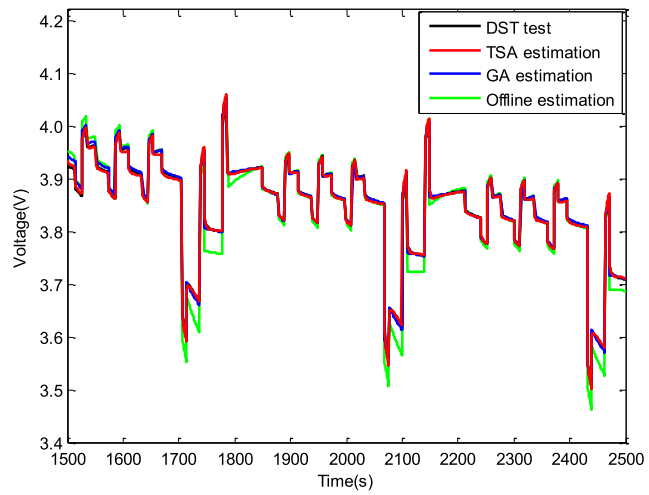

(b)

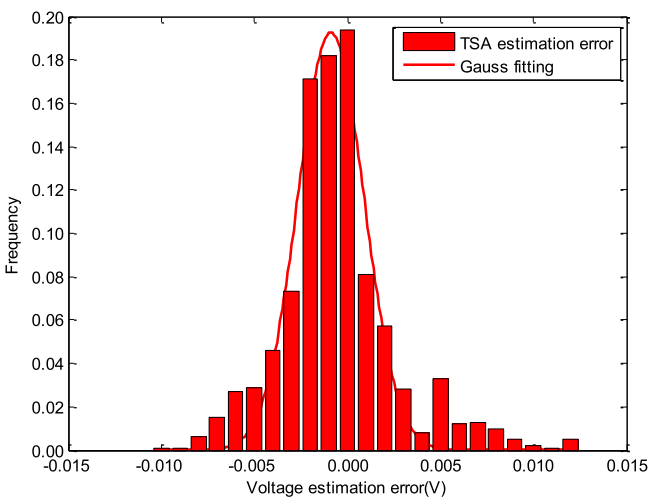

(d)

Fig. 5 Voltage estimation results in the DST test: (a) current and voltage profiles of DST test; (b) voltage estimations of different algorithms; (c) estimation errors of different algorithms; (d) frequency distribution of estimation error with the proposed method. 
Fig. 5 (c). For more intuitive analysis, the error probability distribution of the proposed method is calculated and presented in Fig. 5 (d). The statistic results of different methods are listed in Table 2.

The current and voltage profiles of FUDS test are shown in Fig. 6 (a). The estimated voltages and their errors are demonstrated in Fig. 6 (b) and Fig. 6 (c). The error probability distribution of the proposed method under FUDS test is shown in Fig. 6(d). The statistic results are listed in Table 3 .

From Fig. 5 (b) and Fig. 5 (c), it can be seen that the estimation errors of TSA and GA are smaller than that of the offline approach, indicating that the online identification has a higher precision than the offline approach.

It should be noted that the estimation error of the offline approach could be up to $70 \mathrm{mV}$, which shows that inaccurate parameters may result in unacceptable estimation error. The inaccuracy of the parameters is mainly from the change of current rate. The actual current rate could be up to $4 \mathrm{C}$ in the DST test, but meanwhile it is only about C/3 in HPPC

Table 2 Statistics of voltage estimations in the DST test.

\begin{tabular}{cccc}
\hline Methods & TSA & GA & Offline approach \\
\hline RMSE $(\mathrm{mV})$ & 3.28 & 6.96 & 19.69 \\
MAE $(\mathrm{mV})$ & 12.56 & 27.98 & 70.04 \\
\hline
\end{tabular}
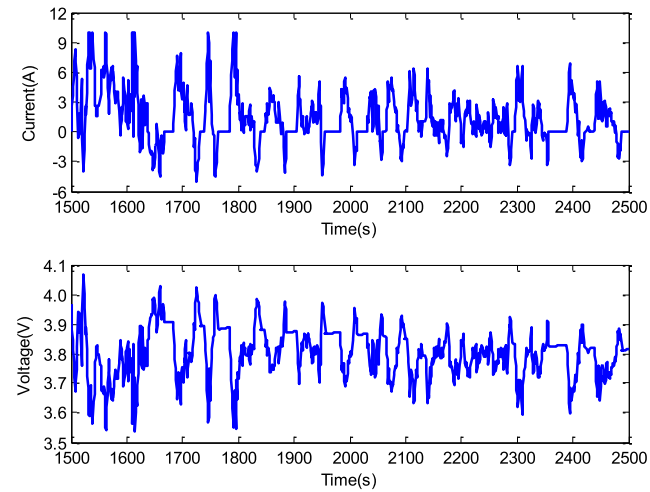

(a)

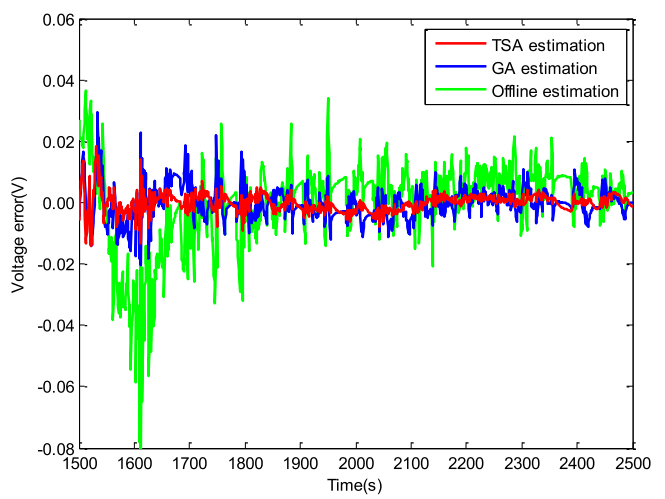

(c) test. To solve the issue, the offline test can be conducted for several times to obtain parameter tables at different rates, but it is time consuming and may lead to significant memory requirement.

By contrast, the online identification methods are able to adapt to real time parameters, and to achieve more accurate results. The error distribution in Fig. 5 (d) shows that the error of the proposed method is mostly located in a region between $-0.010 \mathrm{~V}$ and $0.012 \mathrm{~V}$, which is accurate enough for real time applications. From Table 2 it can be seen that the RMSE and MAE of TSA is $3.28 \mathrm{mV}$ and $12.56 \mathrm{mV}$ respectively, which are obviously smaller than those in GA and the offline method.

From Fig. 6 and Table 3, it can also be seen that the proposed TSA presents an improvement in tracking performance compared with other methods, suggesting that the proposed method is effective and is able to provide more accurate result for parameter identification.

The reference and estimated SOC profiles of DST test are shown in Fig. 7 (a) and the estimation errors are demon-

Table 3 Statistics of voltage estimations in the FUDS test.

\begin{tabular}{cccc}
\hline Methods & TSA & GA & Offline approach \\
\hline RMSE $(\mathrm{mV})$ & 3.06 & 6.32 & 13.63 \\
MAE $(\mathrm{mV})$ & 18.24 & 30.41 & 80.45 \\
\hline
\end{tabular}

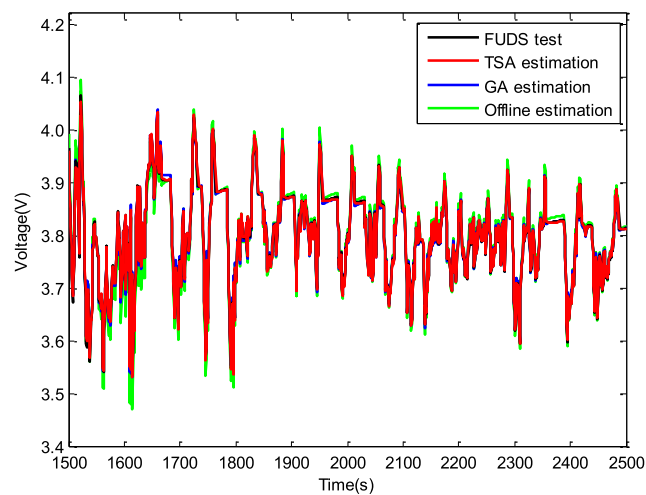

(b)

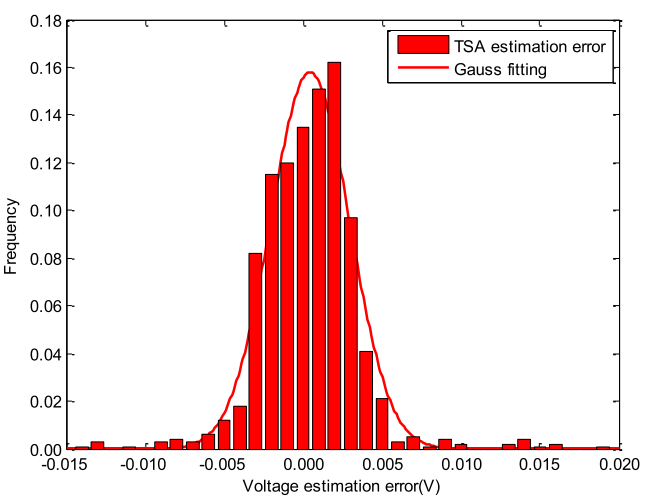

(d)

Fig. 6 Voltage estimation results in the FUDS test: (a) current and voltage profiles of FUDS test; (b) voltage estimations of different algorithms; (c) estimation errors of different algorithms; (d) frequency distribution of estimation error with the proposed method. 


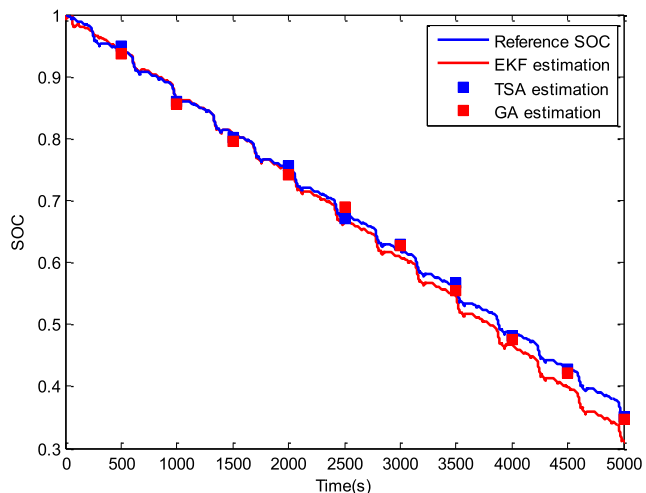

(a)

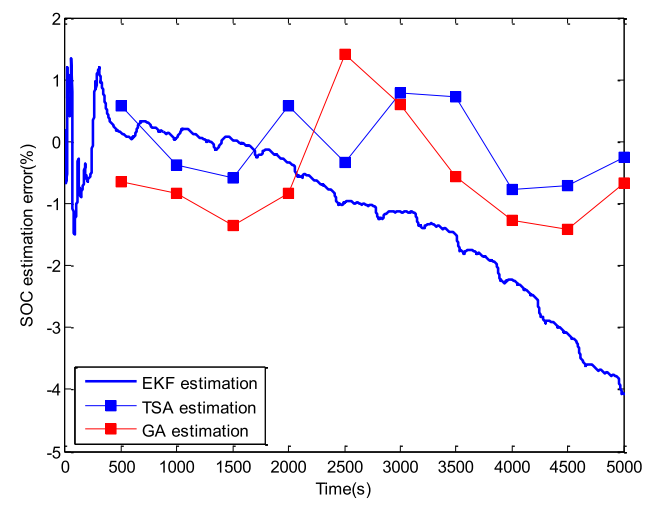

(b)

Fig. 7 SOC estimation results in the DST test: (a) SOC estimations of different algorithms; (b) estimation errors of different algorithms.

Table 4 Statistics of SOC estimations in the DST test.

\begin{tabular}{cccc}
\hline Methods & TSA & GA & Classical EKF \\
\hline RMSE (\%) & 0.60 & 1.01 & 1.67 \\
MAE (\%) & 0.78 & 1.41 & 4.07 \\
\hline
\end{tabular}

strated in Fig. 7 (b). Here the reference real time SOC is calculated by Coulomb counting method using a high precision sensor. The statistic results are listed in Table 4. As to TSA and GA, ten check points are selected for SOC estimation. For each point, the current and voltage data in the previous 1000s are used for estimation. Here the currents are added by a $0.1 \mathrm{~A}$ drift noise to simulate the less accurate sensor in reality application. For comparison, the EKF is introduced to estimate the battery SOC as well, where the parameters are obtained from Table 1. The estimation results under FUDS test are shown in Fig. 8 (a), Fig. 8 (b) and Table 5 .

From Fig. 7 (a) and Fig. 7 (b), it can be seen that the proposed method has a better performance than the two other methods. From Table 4 it can also be seen that the RMSE of TSA is $0.60 \%$. Meanwhile, the RMSE of GA is $1.01 \%$. Such result indicates that the proposed method can achieve better estimation accuracy. To compare the computational efficiency of TSA and GA, the running times are calculated. Here the population sizes of both methods are set to 20 and the generations are fixed to 50 . The running

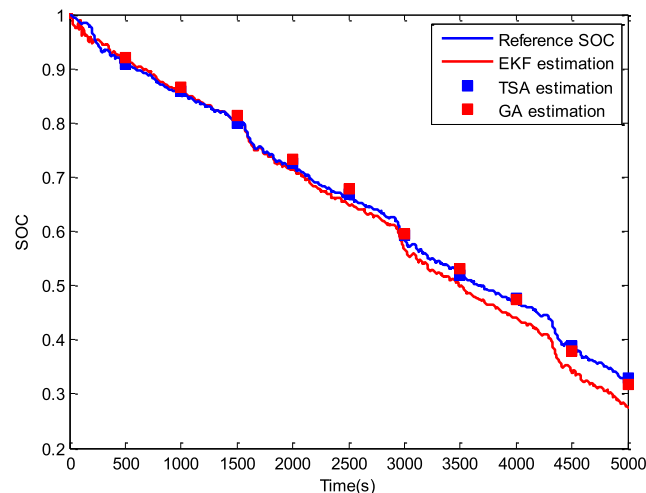

(a)

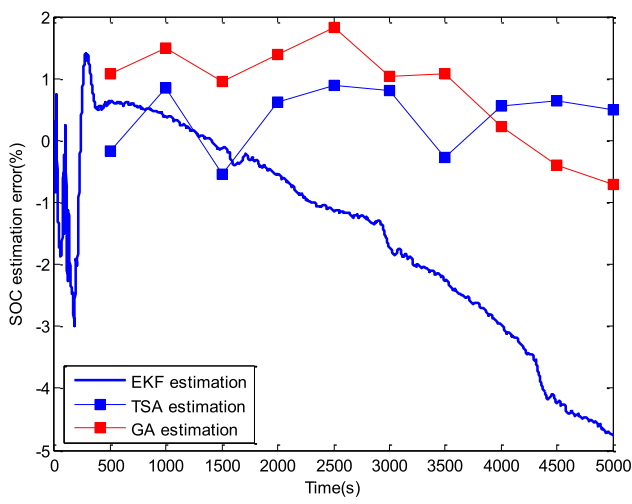

(b)

Fig. 8 SOC estimation results in the FUDS test: (a) SOC estimations of different algorithms; (b) estimation errors of different algorithms.

Table 5 Statistics of SOC estimations in the FUDS test.

\begin{tabular}{cccc}
\hline Methods & TSA & GA & Classical EKF \\
\hline RMSE (\%) & 0.63 & 1.12 & 2.19 \\
MAE (\%) & 0.89 & 1.83 & 4.75 \\
\hline
\end{tabular}

times of TSA and GA in the 1000s DST test are 3.635s and $4.109 \mathrm{~s}$, respectively. Such result shows that the proposed method has higher efficiency than GA.

From Fig. 8 (a), Fig. 8 (b) and Table 5, same conclusions can be drawn. The running time of TSA and GA in the 1000s FUDS test is 3.806 and 4.224 s, respectively. The results indicate that the estimation accuracy and calculation speed can be improved by the propose method. Compared with GA, in which the mutation is mainly caused by small alterations of genes, the proposed method generates mutant vectors by combining different individuals and thus can achieve a higher efficiency. It should be also noted that the estimation error of EKF in both DST and FUDS increase with time and the RMSE and MAE are obviously higher than the other two methods. Such result indicates that the EKF is sensitive to drift noise and the estimation error will be accumulated over time. For the proposed TSA-based method, since the SOC is estimated using a short period of time, the effect of drift noise could be controlled in a limited scope. The estimation errors remain in an acceptable range even if the input data is drifted by noise, which demonstrates 
the robustness of the proposed method.

\section{Conclusion}

In this paper, an enhanced parameter identification method for Li-ion batteries based on an improved tree seed algorithm is proposed. To maintain the diversity of the search candidates, a differential evolution based mutation mechanism is incorporated into the original TSA. To balance the global search and local intensification capabilities of the method, the control parameter is adaptively adjusted during the searching process. An improvement of model accuracy with the proposed method is demonstrated under different dynamic tests. To achieve joint estimation, the battery SOC is included as one of the parameters. Experimental results show that the SOC estimation with improved TSA can yield better performance in terms of accuracy and speed, comparing with GA and EKF. Besides, such method is able to provide SOC estimation without interrupting system operations, and is robust to drift noise disturbance. Therefore it is suitable for online use. Compared with the OCV method, which is widely used as a supplement for Coulomb counting, the proposed method has no need to wait for the battery to be stabilized. Thus it can be used for SOC calibration under both working and charging conditions. Compared with filter based approaches, the model parameters can be updated in real time, and thus the estimation accuracy can be improved. The algorithm will be implemented and verified on BMS under practical conditions in the future.

\section{Acknowledgments}

This work was supported by the National Natural Science Foundation of China (Grant No.11574407) and Science and Technology Planning Project of Guangdong Province, China (2015B010135006, 2017B010120002).

\section{References}

[1] L. Lu, X. Han, J. Li, J. Hua, and M. Ouyang, "A review on the key issues for lithium-ion battery management in electric vehicles," Journal of Power Sources, vol.226, no.3, pp.272-288, 2013.

[2] J. Li, J. Klee Barillas, C. Guenther, and M.A. Danzer, "A comparative study of state of charge estimation algorithms for LiFePO4 batteries used in electric vehicles," Journal of Power Sources, vol.230, pp.244-250, 2013.

[3] G.L. Plett, "Extended Kalman filtering for battery management systems of LiPB-based HEV battery packs: Part 3. State and parameter estimation," Journal of Power Sources, vol.134, no.2, pp.277-292, 2004.

[4] R. Xiong, X. Gong, C.C. Mi, and F. Sun, "A robust state-of-charge estimator for multiple types of lithium-ion batteries using adaptive extended Kalman filter," Journal of Power Sources, vol.243, pp.805-816, 2013.

[5] R. Xiong, H. He, F. Sun, and K. Zhao, "Evaluation on state of charge estimation of batteries with adaptive extended Kalman filter by experiment approach," IEEE Trans. Veh. Technol., vol.62, no.1, pp.108-117, 2013

[6] S. Piller, M. Perrin, and A. Jossen, "Methods for state-of-charge determination and their applications," Journal of Power Sources, vol.96, no.1, pp.113-120, 2001.

[7] W. Waag, C. Fleischer, and D.U. Sauer, "Critical review of the methods for monitoring of lithium-ion batteries in electric and hybrid vehicles," Journal of Power Sources, vol.258, pp.321-339, 2014.

[8] M. Mastali, J. Vazquez-Arenas, R. Fraser, M. Fowler, S. Afshar, and M. Stevens, "Battery state of the charge estimation using Kalman filtering," Journal of Power Sources, vol.239, pp.294-307, 2013.

[9] C. Hametner and S. Jakubek, "State of charge estimation for lithium ion cells: Design of experiments, nonlinear identification and fuzzy observer design," Journal of Power Sources, vol.238, pp.413-421, 2013.

[10] V. Pop, H.J. Bergveld, P.H.L. Notten, and P.P.L. Regtien, "State-of-the-art of battery state-of-charge determination," Measurement Science and Technology, vol.16, no.12, pp.R93-R110, 2005.

[11] P. Tagade, K.S. Hariharan, P. Gambhire, S.M. Kolake, T. Song, D. Oh, T. Yeo, and S. Doo, "Recursive Bayesian filtering framework for lithium-ion cell state estimation," Journal of Power Sources, vol.306, pp.274-288, 2016.

[12] F. Sun, X. Hu, Y. Zou, and S. Li, "Adaptive unscented Kalman filtering for state of charge estimation of a lithium-ion battery for electric vehicles," Energy, vol.36, no.5, pp.3531-3540, 2011.

[13] Z. Zhou, H. Liao, B. Gu, K.M.S. Huq, S. Mumtaz, and J. Rodriguez, "Robust Mobile Crowd Sensing: When Deep Learning Meets Edge Computing," IEEE Netw., vol.32, no.4, pp.54-60, 2018.

[14] Y. Shen, "Adaptive online state-of-charge determination based on neuro-controller and neural network," Energy Conversion and Management, vol.51, no.5, pp.1093-1098, 2010.

[15] S. Grewal and D.A. Grant, "A novel technique for modelling the state of charge of lithium ion batteries using artificial neural networks," 23rd International Telecommunications Energy Conference, Edinburgh, UK, no.484, pp.174-179, 2001.

[16] C. Wu, Z. Liu, D. Zhang, T. Yoshinaga, and Y. Ji, "Spatial Intelligence toward Trustworthy Vehicular IoT," IEEE Commun. Mag., vol.56, no.10, pp.22-27, 2018.

[17] K.T. Chau, K.C. Wu, and C.C. Chan, "A new battery capacity indicator for lithium-ion battery powered electric vehicles using adaptive neuro-fuzzy inference system," Energy Conversion and Management, vol.45, no.11-12, pp.1681-1692, 2004.

[18] P. Singh, R. Vinjamuri, X. Wang, and D. Reisner, "Design and implementation of a fuzzy logic-based state-of-charge meter for li-ion batteries used in portable defibrillators," Journal of Power Sources, vol.162, no.2, pp.829-836, 2006.

[19] S. Malkhandi, "Fuzzy logic-based learning system and estimation of state-of-charge of lead-acid battery," Engineering Applications of Artificial Intelligence, vol.19, no.5, pp.479-485, 2006.

[20] D. Andre, C. Appel, T. Soczka-Guth, and D.U. Sauer, "Advanced mathematical methods of SOC and SOH estimation for lithium-ion batteries," Journal of Power Sources, vol.224, no.5, pp.20-27, 2013.

[21] H. Sheng and J. Xiao, "Electric vehicle state of charge estimation: Nonlinear correlation and fuzzy support vector machine," Journal of Power Sources, vol.281, pp.131-137, 2015.

[22] J.N. Hu, J.J. Hu, H.B. Lin, X.P. Li, C.L. Jiang, X.H. Qiu, and W.S. $\mathrm{Li}$, "State-of-charge estimation for battery management system using optimized support vector machine for regression," Journal of Power Sources, vol.269, pp.682-693, 2014.

[23] X. Han, M. Ouyang, L. Lu, and J. Li, "Simplification of physicsbased electrochemical model for lithium ion battery on electric vehicle. Part II: Pseudo-two-dimensional model simplification and state of charge estimation," Journal of Power Sources, vol.278, pp.814-825, 2015.

[24] X. Hu, F. Sun, and Y. Zou, "Estimation of state of charge of a lithium-ion battery pack for electric vehicles using an adaptive luenberger observer," Energies, vol.3, no.9, pp.1586-1603, 2010.

[25] F. Zhong, H. Li, S. Zhong, Q. Zhong, and C. Yin, "An soc estimation approach based on adaptive sliding mode observer and fractional order equivalent circuit model for lithium-ion batteries," Communications in Nonlinear Science and Numerical Simulation, vol.24, 
no.1-3, pp.127-144, 2015 .

[26] G.L. Plett, "Extended Kalman filtering for battery management systems of LiPB-based HEV battery packs: Part 2. Modeling and identification," Journal of Power Sources, vol.134, no.2, pp.262-276, 2004.

[27] G.L. Plett, "Sigma-point Kalman filtering for battery management systems of LiPB-based HEV battery packs: Part 2: Simultaneous state and parameter estimation," Journal of Power Sources, vol.161, no.2, pp.1369-1384, 2006

[28] M. Coleman, W.G. Hurley, and C.K. Lee, "An improved battery characterization method using a two-pulse load test," IEEE Trans. Energy Convers., vol.23, no.2, pp.708-713, 2008.

[29] U. Tröltzsch, O. Kanoun, and H.-R. Tränkler, "Characterizing aging effects of lithium ion batteries by impedance spectroscopy," Electrochimica Acta, vol.51, no.8-9, pp.1664-1672, 2006.

[30] D. Andre, M. Meiler, K. Steiner, H. Walz, T. Soczka-Guth, and D.U. Sauer, "Characterization of high-power lithium-ion batteries by electrochemical impedance spectroscopy. II: Modelling," Journal of Power Sources, vol.196, no.12, pp.5349-5356, 2011.

[31] A. Singh, A. Izadian, and S. Anwar, "Model based condition monitoring in lithium-ion batteries," Journal of Power Sources, vol.268, pp.459-468, 2014.

[32] H. He, X. Zhang, R. Xiong, Y. Xu, and H. Guo, "Online modelbased estimation of state-of-charge and open-circuit voltage of lithium-ion batteries in electric vehicles," Energy, vol.39, no.1, pp.310-318, 2012.

[33] G. Dong, Z. Chen, J. Wei, C. Zhang, and P. Wang, "An online model-based method for state of energy estimation of lithium-ion batteries using dual filters," Journal of Power Sources, vol.301, pp.277-286, 2016

[34] J. He and Z. Hou, "Ant colony algorithm for traffic signal timing optimization," Advances in Engineering Software, vol.43, no.1, pp.14-18, 2012.

[35] N. Li, "A mathematical model of urban hazardous chemicals logistics distribution route decision based on improved genetic algorithm," Chemical Engineering Transactions, vol.71, pp.481-486, 2018.

[36] Z. Zhou, L. Tan, B. Gu, Y. Zhang, and J. Wu, "Bandwidth Slicing in Software-Defined 5G: A Stackelberg Game Approach," IEEE Veh. Technol. Mag., vol.13, no.2, pp.102-109, 2018.

[37] M. Ouyang, G. Liu, L. Lu, J. Li, and X. Han, "Enhancing the estimation accuracy in low state-of-charge area: A novel onboard battery model through surface state of charge determination," Journal of Power Sources, vol.270, pp.221-237, 2014.

[38] T. Hu, B. Zanchi, and J. Zhao, "Simple analytical method for determining parameters of discharging batteries," IEEE Trans. Energy Convers., vol.26, no.3, pp.787-798, 2011.

[39] S.E. Li, B. Wang, H. Peng, and X. Hu, "An electrochemistry-based impedance model for lithium-ion batteries," Journal of Power Sources, vol.258, pp.9-18, 2014.

[40] M.A. Rahman, S. Anwar, and A. Izadian, "Electrochemical model parameter identification of a lithium-ion battery using particle swarm optimization method," Journal of Power Sources, vol.307, pp.86-97, 2016.

[41] J. Feng, Z. Liu, C. Wu, and Y. Ji, "AVE: Autonomous vehicular edge computing framework with ACO-based scheduling," IEEE Trans. Veh. Technol., vol.66, no.12, pp.10660-10675, 2017.

[42] J. Feng, Z. Liu, C. Wu, and Y. Ji, "Mobile Edge Computing for the Internet of Vehicles: Offloading Framework and Job Scheduling," IEEE Veh. Technol. Mag., vol.14, no.1, pp.28-36, 2019.

[43] M.S. Kiran, "TSA: Tree-seed algorithm for continuous optimization," Expert Systems with Applications, vol.42, no.19, pp.6686-6698, 2015.

[44] X. Li and M. Yin, "Parameter estimation for chaotic systems by hybrid differential evolution algorithm and artificial bee colony algorithm," Nonlinear Dynamics, vol.77, no.1-2, pp.61-71, 2014.

[45] N. Omar, M. Daowd, O. Hegazy, G. Mulder, J.-M. Timmermans, T.
Coosemans, P.V. Bossche, and J.V. Mierlo, "Standardization work for BEV and HEV applications: Critical appraisal of recent traction battery documents," Energies, vol.5, no.1, pp.138-156, 2012.

[46] C. Zhang, K. Li, L. Pei, and C. Zhu, "An integrated approach for real-time model-based state-of-charge estimation of lithium-ion batteries," Journal of Power Sources, vol.283, pp.24-36, 2015.

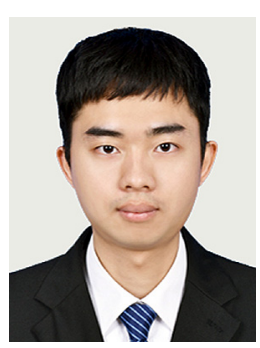

Weijie Chen received his B.S. degree in School of Engineering from Sun Yat-sen University, Guangzhou, China, in 2012, where he is currently pursuing the $\mathrm{Ph} . \mathrm{D}$. degree from School of Intelligent Systems Engineering, Sun Yat-sen University. His current research interests include nonlinear system identification, optimizing algorithms and their applications in electric vehicles.

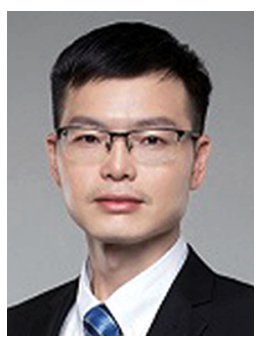

Ming Cai received his Ph.D. degree from Sun Yat-sen University in 2004. He is now a professor at School of Intelligent Systems Engineering, Sun Yat-sen University. He has been in charge of National Science-technology Projects and has published over 30 academic papers index by SCI/EI journals. His researches mainly focus on intelligent transportation system, traffic environment and traffic signal optimization.

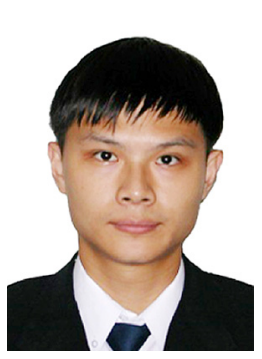

Xiaojun Tan received his Ph.D. degree from Sun Yat-sen University in 2005. He is now an associate professor at School of Intelligent Systems Engineering, Sun Yat-sen University. He has published two technical monographs and applied over 30 patents. His researches mainly focus on electric vehicles, battery management system and automatic control system.

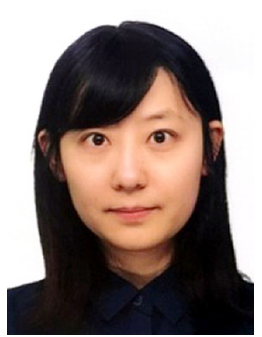

Bo Wei received the B.E. and M.E. degrees from Tianjin University, Tianjin, China, in 2012 and 2015, respectively. She is currently working toward the Ph.D. degree at the Graduate School of Fundamental Science and Engineering, Waseda University. She is a student member of the IEEE and IEICE. 